\title{
Message in a bottle
}

Many bacterial species release extracellular vesicles, which have roles in various processes, including quorum sensing, virulence and horizontal gene transfer. Chisholm and colleagues now report the discovery that the dominant marine cyanobacterium, Prochlorococcus spp., produces an abundance of extracellular vesicles, which might have diverse functions in the ocean ecosystem and affect carbon cycling.

Extracellular vesicles are produced by various cells; in Gram-negative bacteria, parts of the outer membrane are pinched off, which leads to the release of vesicles that contain cellular lipids and proteins. When studying scanning electron micrographs of Prochlorococcus spp., the authors observed numerous small spherical structures that resembled extracellular vesicles, close to the cell surface. Transmission electron microscopy and nanoparticle-tracking analysis

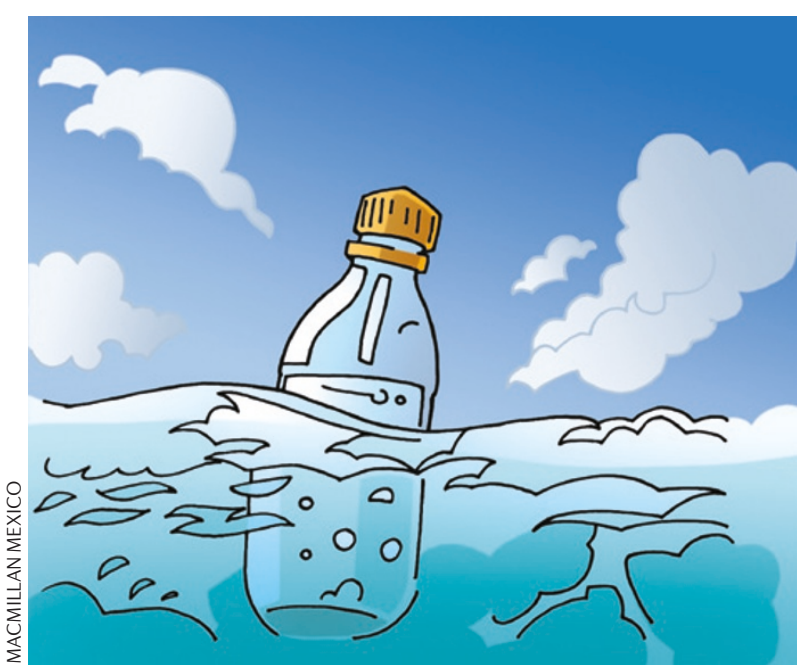

of cultured Prochlorococcus spp. confirmed that the particles were extracellular vesicles. The authors found vesicles in cultures from all six Prochlorococcus strains that they studied.

The vesicles ranged in size from $70 \mathrm{~nm}$ to $100 \mathrm{~nm}$ and contained lipids, proteins and nucleic acids that matched the producer bacteria; they contained not only membrane proteins and lipids but also cytoplasmic proteins, DNA fragments that covered more than $50 \%$ of the genome and RNA from $95 \%$ of all open reading frames. On the basis of the number of vesicles found in the cultures, the authors estimated that wild Prochlorococcus cells release $10^{27}-10^{28}$ vesicles, and thus many cellular proteins and lipids, into the global oceans each day, which would amount to at least $10^{4}-10^{5}$ tonnes of fixed carbon.

The marine environment is limited in nutrients, so why would Prochlorococcus spp. potentially waste the carbon, nitrogen and phosphorus that are fixed in cellular components by releasing them in vesicles? The authors propose several possible functions for the secreted vesicles. First, vesicles might function as gene transfer vehicles; indeed, the vesicles contain fragments of Prochlorococcus DNA, some of which are at least $3 \mathrm{~kb}$ in size, which would be large enough to accomodate multiple genes. Second, the nutrients that are contained in the vesicles might promote the growth of 'helper' bacteria that are advantageous for Prochlorococcus spp. - for example, by the enzymatic neutralization of reactive oxygen species, which is a function that Prochlorococcus spp. lack. Indeed, isolated vesicles were sufficient as the sole carbon source to support the growth of marine heterotrophic bacteria. Third, as the vesicles contain many Prochlorococcus surface proteins to which phages can bind, it is possible that they function as decoys to adsorb phages in the surrounding seawater, thereby reducing attacks on bacterial cells. Interestingly, transmission electron micrographs showed phages with shortened stalks and altered capsid staining bound to vesicles, which indicates that they had injected their DNA. These are all intriguing possibilities for the potential functions of Prochlorococcus vesicles, but further work is needed to clarify their exact roles in the marine environment.

Nevertheless, it is clear that extracellular vesicles are not only important only for Prochlorococcus spp., as the authors also detected vesicles in cultures of another marine bacterium, Synechococcus strain WH8102, and in two seawater samples from the Massachusetts coast and the Sargasso Sea, in equal abundance to bacterial cells and containing DNA from many bacterial phyla. Vesicle secretion might be a widespread mechanism for the transport of various molecules in the marine ecosystem.

Ursula Hofer

ORIGINAL RESEARCH PAPER Biller, S. J. et al. Bacterial vesicles in marine ecosystems. Science 343, 183-186 (2014) 